Oikos 116: 1247-1253, 2007

doi: $10.1111 /$ j.2007.0030-1299.15686.x,

Copyright (C) Oikos 2007, ISSN 0030-1299

Subject Editor: Tim Benton, Accepted 9 March 2007

\title{
Publication bias and merit in ecology
}

\author{
C. J. Lortie, L. W. Aarssen, A. E. Budden, J. K. Koricheva, R. Leimu and T. Tregenza \\ C. J. Lortie (lortie@yorku.ca), Dept of Biology, York Univ., 4700 Keele St., Toronto, Ontario, Canada, M6S 2E2. - L. W. Aarssen, \\ Dept of Biology, Queen's Univ., Kingston, ON, Canada, K7L 3N6. - A. E. Budden, Dept of Ecology and Evolutionary Biology, Univ. \\ of Toronto, Toronto, ON, Canada, MSS 3B2. - J. K. Koricheva, School of Biological Sciences, Royal Holloway, Univ. of London, \\ Egham, Surrey, UK, TW20 OEX. - R. Leimu, Inst. for Biochemistry and Biology, Univ. of Potsdam, Maulbeerallee 1, DE-14469 \\ Potsdam, Germany. - T. Tregenza, Centre for Ecology and Conservation, Univ. of Exeter, Cornwall Campus, Tremough, Penryn, \\ UK, TR10 9EZ.
}

\begin{abstract}
Bias, or any set of factors that influence the general expression of merit, is common in science and is an inevitable by-product of an imperfect but otherwise reasonably objective human pursuit to understand the world we inhabit. In this paper, we explore the conceptual significance of a relatively tractable form of bias, namely publication and dissemination bias. A specific definition is developed, a working model of classification for publication bias is proposed, and an assessment of what we can measure is described. Finally, we offer expectations for ecologists with respect to the significance of bias in the publication process within our discipline. We argue that without explicit consideration of both the qualitative and quantitative aspects of publication bias in ecology, we limit our capacity to fairly assess and best use the science that we as a community produce.
\end{abstract}

It is now recognized that biases function at many levels within science including funding allocation, employment, publication, and general research directions. While misrepresentation can be described very broadly as 'bias' and in some cases may result from misconduct, bias can occur as an incidental product of entirely legitimate practices. In the latter case, there may be no contempt warranted for bias. Nonetheless, we must address indifference to bias by analysing its potential effects on the practice and progress of science. A few recent examples highlight the problems that are facing the scientific community. For instance, the 'leaky pipeline' is a potential form of gender bias where, although equal numbers of men and women enter science, significantly fewer women obtain full professor status (Schubert and Sinha 2004). Explanations include potential changes in ambition levels by women due to the nature of the graduate academic environment (Sears 2003) or differential success in securing doctoral or post-doctoral funding (Bornmann and Daniel 2005). More proximate examples include differences between journals (in ecology and evolution) in the rejection rates of papers by gender (Tregenza 2002) or an alarming lack of female respondent authors (6\% of a total of 151 ecologists) in a major poll of reasons for rejection of papers (Cassey and Blackburn 2003, 2004). Preliminary evidence for general biases in the publication process independent of gender include differential citation frequency based on initial letter of surname (Tregenza 1997), individuals with more previous publications having lower percentages of articles accepted on first submission (Cassey and Blackburn 2004), and biases where authors choose to submit papers based on the statistical significance of their studies (Koricheva 2003). There are many explanations for these coarse findings; however, it is reasonable to suggest that ecologists should explicitly consider and define areas of concern if not consider changing our scientific practices given appropriate inspection. Impact factors of journals also suffer from bias (at least in a statistical sense) in that the distribution of citation rates of articles within a journal can be highly skewed (Seglen 1994, Colquhoun 2003) meaning that 'bad' papers are frequently found in good journals and vice versa. Furthermore, impact factor 
varies between disciplines because it is simply a product of the number of citations per paper and the age distribution of cited papers within a discipline. Factors such as the size of a particular field or indeed its quality are not typically reflected in impact factors (Kokko and Sutherland 1999). This cursory narrative review leads us to propose that there is a definitive need to examine most forms of bias in ecology, from people to papers. A logical concrete starting point however, and the premise for this paper, is to explore one of the more evident and tangible products of ecological research - peer-reviewed publications. Increasingly, total number of publications and citation frequency are metrics widely used in ecology to evaluate the merit of science and of scientists (Adam 2002). However, only very limited direct evidence is available on the appropriateness or context of use for these measures. As such, inspection of the publication and dissemination process is relevant not only to the products we offer to the general community and how they are viewed but to the benchmarks of individual success and the composition of our working field.

Although studied extensively in the medical sciences (now with five international congresses on peer review and biomedical publication - see http://www.ama-assn. org/public/peer/peerhome.htm), publication bias has only recently started to receive attention among ecologists (Alatalo et al. 1997, Tregenza and Wedell 1997, Kotiaho 1999b, Palmer 1999, Duvall and Tweedie 2000, Møller and Jennions 2001, Jennions and Møller 2002, Tregenza 2002, Cassey and Blackburn 2004, Leimu and Koricheva 2004, 2005a, 2005b, Tomkins and Kotiaho 2004). To develop an appropriate conceptual basis for subsequent quantitative tests for publication bias, we develop the following necessary components: a definition of publication bias based on merit, a working model for classification, an assessment of measurement approaches, and a set of expectations for ecologists with respect to the dissemination process.

\section{Defining publication bias by merit}

Publication is both a process and a medium that scientists use for the transmission and dissemination of knowledge and ideas. A publication, defined in the broadest sense, is generated when a manuscript is presented to an audience and is justified by its likelihood of future use by others. Indicators of use may include feedback from colleagues, reviewers' comments, an editor's acceptance (or rejection), the status of the journal for publication, download statistics from on-line journals, and citation rate. We generally assume that usage reflects merit, but what if papers are used by others for reasons not related to the science itself? Perception is integral to science by not only informing what we decide to test but also how we use the ideas of others. Therefore, scientific merit is more accurately modelled by relative perceived merit (RPM). Merit requires use at some level, and peer-reviewed publications are the key medium to disseminate ideas and data. The definition of merit through perception emphasizes that the RPM of studies not submitted and hence unpublished is for all intents and purposes not measurable. Conversely, as a given published idea matures, it should accumulate merit through realized use at some rate that can sometimes reflect its perceived merit since human judgement is subject to error.

We suggest that the magnitude of this error at least in part represents publication and dissemination biases. Does relative perceived merit accurately represent relative actual merit (RAM), i.e. the scientific merit that a paper is entitled to based on first principles? The extent to which RPM is unable to predict RAM is a measure of bias. A perfect fit occurs when the regression is proportional to an $r^{2}$ of 1.0 (Fig. 1a). 'Systemic' bias occurs across the discipline if the relationship is disproportional (Fig. 1a). An individual paper incurs 'local' publication bias relative to others in the discipline when its regression residual departs from zero, either negatively or positively, representing underor over-representation respectively (Fig. 1b). Previous definitions of bias generally describe the tendency for researchers to submit and/or for reviewers to accept only papers that have positive outcomes or large effect sizes (Table 1). We propose here that publication bias is best defined more inclusively as the degree of divergence between the relative actual merit of a given publication and its relative perceived merit.

While relative actual merit, not unlike evolutionary fitness, can be understood and defined, it cannot be measured directly. We can expect the relationship between RPM and RAM to be positive (Fig. 1), but we can only test for indications of potential bias. For example, we may show that collaboration with an established, highly cited co-author increases one's citation rate, but without experimental manipulation we cannot determine whether this is due to the addition of the collaborator in name only (RPM) or a consequence of high quality contribution from the collaborator (RAM). If readers cite a paper primarily because it includes a famous author, rather than due to its merit, then a poor fit between RPM and RAM occurs, demonstrating bias. On the other hand, if readers cite a paper only because of its content, and if merit genuinely is greater if the paper includes an established, experienced co-author, then there is no publication bias. These translation errors of merit into bias permit us only to infer bias in a very coarse manner until we better refine the process of dissemination and recognition of merit in ecology. Of course, there are also simple measurement errors, general tendencies to undervalue 

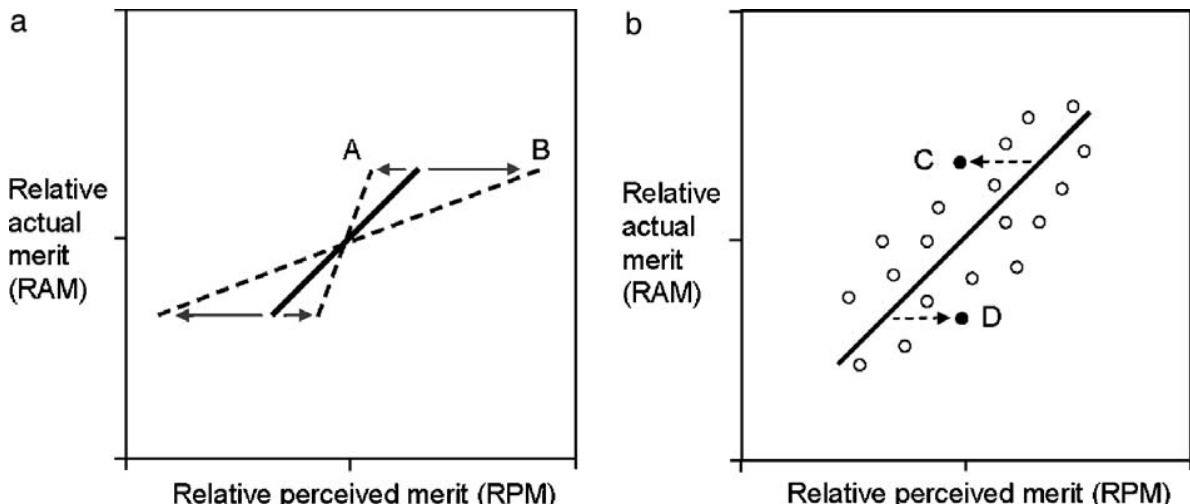

Fig. 1. When perceived merit of a publication perfectly predicts actual merit, then there is no bias. This occurs when the relationship is perfectly proportional (regression slope $=1.0$ ) and regression $r^{2}=1.0$ (solid black line in panel (a)). Publication bias may be manifested in two ways: (a) Systemic bias across the whole discipline is evident when the regression slope $>1.0$, implying a narrower variance for perceived merit than for actual merit (line A), or when the slope $<1.0$, implying a wider variance for perceived merit than for actual merit (line B). Note that actual merit, by definition, has no error, and so it's range is fixed; (b) When slope $=1.0$ (i.e. the relationship is proportional), there is still local bias when the regression $\mathrm{r}^{2}<1.0$. In this case, the level of bias incurred by a particular paper is indicated by the residual (arrows in panel (b)), where actual merit may be underrepresented (point C) or over-represented (point D) by perceived merit.

our work in ecology, and nonlinear deviations in the assignment of merit depending on the initial RPM of the paper. For the purposes of improving how we do ecology and how we assign value to publications, we assume that potential publication bias, or the suite of factors that generate deviation of perceived from actual, can be deconstructed into tests of the relative importance of attributes associated with a publication other than its intrinsic worth. The most relevant deviations are not those that are spurious but those that can be related to other factors we can and do define such as gender, nationality, or any other attribute we might perceive as important. An indicator of potential publication bias is thus identified when a particular factor (e.g. author gender) is shown to have a significant relationship with perceived merit (e.g. paper rejection rate, citation rate) when there is no objective, a-priori reason to expect that it should.

\section{Classifications of potential publication bias}

To enhance the capacity of ecologists to quantitatively assess bias and to develop a working contextual model of causation, we have identified three levels of the publication and dissemination process; namely, characteristics of the study (number of hypotheses,

Table 1. A list of the current definitions for publication-related biases. The definition adopted by Gurevitch and Hedges (1999) is representative of the most common usage in the meta-analysis literature while the definition of Bornmann and Daniel (2005) is common in the medical literature.

\begin{tabular}{llc}
\hline Citation & Term & Definition
\end{tabular}

\section{Csada et al. 1996}

Gurevitch and Hedges 1999

Song et al. 2000

Møller and Jennions 2001
Publication bias Publication bias

Publication bias

Dissemination bias

Bias

Publication bias

Submission bias

Bias
Bias in the selection of results for publication.

Publication selection is the tendency for results that are statistically significant to be more likely to be published than those that fail to detect significance.

Publication of research results depends on the direction or strength of findings.

Accessibility of research results depends on the direction and strength of findings.

Systematic deviation of results from the truth, or processes leading to such deviation.

Bias owing to the influence of research findings on submission, review and editorial decisions.

Bias owing to the influence of research findings on the probability of submission.

Judgements not solely based on scientific merit, but are also based on attributes of the authors. 
effect size, support for main hypothesis), attributes of the publication itself (length, number and gender of authors), and attributes of the journal (reputation, impact factor, circulation) (Fig. 2) all of which could be subject to inaccurate assessment of merit, i.e. biases. This list is not entirely comprehensive but provides an indication of the functional attributes that ecologists may use as the substrate to assign merit to a study, for better or worse. It also includes previous delineations of publication bias as submission, editorial, and reviewer biases that can act on the attributes listed at several levels (Møller and Jennions 2001). However, the effects of publication biases likely do not end with the acceptance/rejection of a study but rather interact with and cascade to other dissemination related effects such as citation frequency or establishment of dominant ideas. It would be unrealistic to assume that ecologists do not look at the journal where a study resides, where the study was done and by whom, and whether the hypotheses were supported or rejected when we assign merit to the publication and decide whether we agree or disagree with the findings.

There are also two higher-order properties evident in the expression of all publication biases. Firstly, there are relational effects between the attributes of a study/ publication at the different hierarchical levels (Fig. 2, as depicted by the dotted arrows between the three levels). For instance, simple relationships such as the number of statistical tests and graphics (related to amount of data) positively predicting the length of the paper (in some cases contingent upon journal requirements) or the magnitude of the effect sizes within the publication relating to the type of study (mensurative vs highly controlled manipulative experiment). Testing for these relational effects will help us understand how we as ecologists disseminate our findings and knowledge, and it would be insightful to know whether some subdisciplines communicate similar statistical content differently, i.e. plant ecologists use less graphs, more tables, and write longer papers as compared to

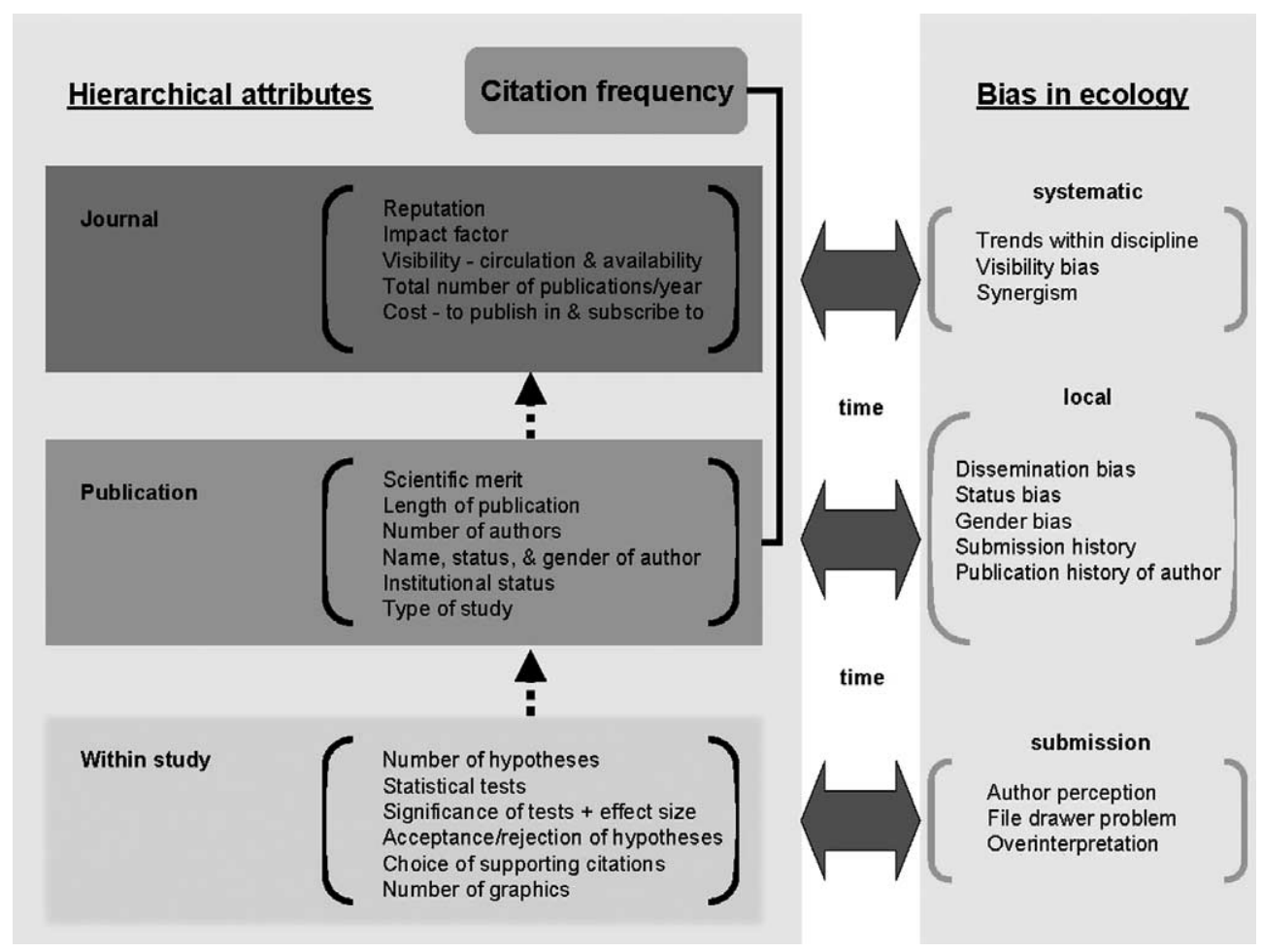

Fig. 2. A conceptual illustration of the publication and dissemination process in ecology with potential publication related biases listed to the right. Attributes at each level of the process are listed as within study (i.e. attributes of the study itself and subsequent interpretations by the author), publication level (properties of the published study), and journal level (associated attributes of the journal wherein a given study is published). Citation frequency refers to the number of times a particular publication is referenced in other publications; however, the impact factor attribute of a journal is also calculated using mean citation frequency of all papers published therein within the last two years and also best conceptualised as an emergent property. The biases listed are generally recognized within ecology and sometimes quantitatively analyzed and published. Nonetheless, few analyses have explored either the potential relationships between attributes of the publication process, as denoted by the dotted arrows, or between the attributes of the process and the various biases which may influence relative success or performance of a study/ publication, as denoted by the double-headed arrows. 
behavioural or molecular ecologists perhaps. Detection of these relationships would help us develop appropriate research questions about the process of ecology as a science versus the process of dissemination. Furthermore, we could compare ecology to the medical or physical sciences and test whether it is data, experimental design, or culture that dictates dissemination practices. More pressing dissemination-related factors to test in ecology clearly include defining relationships between the number and gender of authors and the impact factor of the journal in which a study is published. Correlation is not causation, but testing for these relationships challenges the dominant view in ecology - that successful publication and citation frequency are based solely on scientific 'merit' as defined by first principles in science, and not other attributes of the dissemination process.

The second higher-order attribute evident in this ontology is that bias and the publication attributes can feedback into the dissemination process (Fig. 2, solid double-headed arrows). In some instances, author perception and decisions may lead to RPM that underestimates RAM because of ill-chosen style or cosmetic factors that do not relate the true value of the ideas or discoveries. For example, a title that is not 'catchy' enough or does not use the word 'novel' in the title or abstract (Friedmann and Karlsson 1997), unattractive figures, text that is wordy or pedantic to be fully persuasive, poor English or publication in another language (Nylenna et al. 1994), or language that is insufficiently articulate to capture a large mass of readers' attention. In the most extreme cases, the author may even perpetuate the file-drawer problem by assigning a very low perceived merit to studies with non-significant results and submit only studies with statistically significant results (Csada et al. 1996). Conversely, the attributes within the publication or study may also be subject to bias because RPM overestimates RAM when the author interprets weakly significant results favourably to either increase the likelihood of successful publication or subconsciously because he/she believes in the ideas tested and may be influenced by other observational data not included in the publication (Lortie and Dyer 1999, Lortie 2000). In all these instances, bias is not necessarily an external force selectively driving certain papers through the dissemination pipeline but active choices by the authors which can perpetuate certain tendencies within the scientific community. These author-related decisions likely interact with accurate interpretations of data that challenge a favoured theory and in some instances reduce the likelihood of successful publication or recognition (Campanario 1993, 1995, 1996). These forms of 'interactive' bias set the stage for other sets of factors that might influence the accurate assessment of merit of a publication such as a timing bias - less statistically significant results are delayed in timing of publication or published in low-circulation journals (Song et al. 2000), institutional status bias - authors from higher status institutions have increased ease in publishing (Cassey and Blackburn 2004), visibility bias - journals with higher impact factors are also more visible (Koricheva 2003), and co-author reputation (Leimu and Koricheva 2005a).

Some forms of bias might occur solely due to spurious effects such as 'alphabetical discrimination' which occurs when earlier author surname initials are more highly cited than later positioned surnames in the alphabet (Tregenza and Wedell 1997, Einav and Yariv 2006) or difficulty with non-English surnames (Kotiaho 1999a, 1999b). Unfortunately, these examples illustrate that even scientists are subject to visibilityrelated effects such as scrolling within databases to select all citations instead of the first few to support our statements and ability to type in an unfamiliar name. In some instances, it is reasonable to assume that the attributes are accurate indicators of relative perceived merit such as number of hypotheses (i.e. we might assume that good science sets up contrasting hypotheses with viable alternatives), the type of statistical tests, and perhaps the effect sizes the study generates. However, in other instances, it is not obvious why number of authors, gender, surname, or institutional status would matter. There is also the reality to consider that the attributes and the bias interact (as denoted by the double-headed arrows in Fig. 2) in that selection processes through bias generate feedback loops and either open or close the door for certain sets of ideas, or unfortunately individuals, based on prior performance of papers submitted and publications, thereby shaping the culture, people, and ideas that come to dominate in ecology. To move forward more equitably and mindfully, ecologists must now begin to specifically test research questions and predictions with respect to the dissemination process. If the overarching null hypothesis that publication and citation frequency are based solely on scientific 'merit' is not supported, we must identify the alternative hypotheses that shape the character and culture of practice within our discipline.

\section{Measurement of publication bias}

The primary challenge of quantifying publication bias is to identify the appropriate response variables that represent the true effect of interest while measuring the covariates that may influence respective trends such as status of the author and citation frequency. Using our ontological approach in defining publication bias (Fig. 2), it is clear that many covariates are worth testing with respect to the RPM of a given publication. Firstly, we may test whether the attributes of the dissemination 
process are best viewed as hierarchical, i.e. can we use lower-level attributes to predict higher-level attributes, and if so, explore explanations (dotted arrows in Fig. 2). Secondly, we may use common measures of publication performance such as citation rate of the paper to test for publication biases at all three levels, i.e. submission, local, and systematic biases (solid arrows in Fig. 2). Under this approach, we may additionally test whether time relates to the strength of potential biases (Abt 1998, Van Raan 1999). Thirdly, we may compare the statistical relationships identified in different subdisciplines of ecology to determine whether biases in the dissemination process are general effects or specific to ecological societies, study organisms, or journals. In all three sets of tests, standard correlation and regression techniques including multiple logistic regression and meta-analysis (with funnel plots or trim and fill analyses) and cumulative meta-analysis are excellent tools to begin to test for bias (Møller and Jennions 2001, Leimu and Koricheva 2004). Comparing subdisciplines may require a bit more finesse such as analyses of the rate of change or slope of citation frequency between different types of publications or between different subdisciplines, comparing uneven changes in rejection rates within subdisciplines, using a control variable approach where the performance of the individual scientist or even subdiscipline are also tested as independent variables (Cole and Fiorentine 1991), or invoking synthetic integrative concepts to evaluate RPM such as those developed by Ford (2000). These synthetic concepts could include rankings of acceptability of propositions by data, consistency of definitions with a subdiscipline, consistency in concepts used, avoidance of ad hoc explanations, economy of propositions used, and that explanations generated by a publication apply to broad questions. This approach was successfully applied to testing the efficacy of the peer-review system on graduate-level funding by testing reliability, fairness, and predictive validity of the decisions by a granting agency (Bornmann and Daniel 2005). Ecologists could similarly begin to analyze bias by subdiscipline. It is time to quantitatively explore how the dissemination process assigns merit and examine the assumptions used to interpret ecological data.

\section{Context and expectations for ecologists}

Ecologists have expressed concern that progress in ecology is insufficient (Wilkinson 1998, Austin 1999, O'Connor 2000, Swihart et al. 2002). While we do not argue that there is a lack of progress per se or that ecology should be set to the standards of other disciplines (Aarssen 1997), it is nonetheless valid to question whether there are general limitations or difficulties that influence the success of ecology as an explanatory and predictive discipline of science. One such avenue of progress is the development of theory within ecology which can sometimes be driven by personal research agendas, theory tenacity, or general trends within a particular subdiscipline (Loehle 1987, Aarssen 1997, 1999, Austin 1999). Others have proposed that dissatisfaction may arise due to either unrealistic theory development, oversimplifications of tests of theory, or inadequate approaches applied to assessing progress (Stamp 2003). In our opinion, the most cogent argument to consider when evaluating progress is that of the importance of various biases in ecology (Koricheva 2003). In this paper, we proposed both a conceptual and empirical framework to consider bias in ecology. Scientometrics and other disciplines are rapidly developing tools that can be adapted to study the process of dissemination in ecology. Many aspects of the ecological community deserve consideration, but we propose here that a reasonable starting point for ecologist to begin this introspection, based on our analytical skill base, is to explore publications directly through the quantitative examination of relationships between studies, publications, the journals, and other attributes that might influence the success of papers but should not. Ecology may be unique in some respects since our research focus is diverse and often on highly dynamic natural events and patterns. In the event that publication bias is evident, ecologists can find solutions in place within other disciplines such as double-blind review systems, tighter controls on reviewer selection, i.e. author-suggested versus editor-selected reviewers (Grimm 2005), reviewer training (such as that of $\mathrm{BMJ}$ ), pre-submission independent data-registry, institutes such as the Cochrane Collaboration to externally explore bias, positive discrimination strategies for target groups or individuals, and higher data quality and metadata assessment and documentation procedures associated with publications. At the very minimum, we can expect that this exercise will allow us to identify the filters we use to quickly process the overwhelming amount of literature published and highlight the relative standards we adopt when assessing merit.

Acknowledgements - This work was conducted as part of the 'Role of publication-related biases in ecology' Working Group supported by the National Center for Ecological Analysis and Synthesis, a Center funded by NSF (Grant no. DEB-0072909), the Univ. of California, and the Santa Barbara campus.

\section{References}

Aarssen, L. W. 1997. On the progress of ecology. - Oikos 80: 177-178.

Aarssen, L. W. 1999. Progress drive from progress pride. - Oikos 85: 375-376. 
Abt, H. A. 1998. Why some papers have long citation lifetimes. - Nature 395: 756-757.

Adam, D. 2002. The counting house. - Nature 415: $726-$ 729.

Alatalo, R. V. et al. 1997. Heritabilities and paradigm shifts. - Nature 385: 402-403.

Austin, M. P. 1999. A silent clash of paradigms: some inconsistencies in community ecology. - Oikos 86: 170178.

Bornmann, L. and Daniel, H. D. 2005. Selection of research fellowship recipients by committee peer review. Reliability, fairness, and predictive vailidity of Board Trestees' decisions. - Scientometrics 63: 297-320.

Campanario, J. M. 1993. Consolation for the scientist: sometimes it is hard to publish papers that are later highly cited. - Social Stud. Sci. 23: 342-362.

Campanario, J. M. 1995. Commentary on influential books and journal articles initially rejected because of negative referees' evaluations. - Sci. Comm. 16: 304-325.

Campanario, J. M. 1996. Have referees rejected some of the most-cited papers of all times? - J. Am. Soc. Inf. Sci. 47: 302-310.

Cassey, P. and Blackburn, T. M. 2003. Publication rejection among ecologists. - Trends Ecol. Evol. 18: 375-376.

Cassey, P. and Blackburn, T. M. 2004. Publication and rejection among successful ecologists. - Bioscience 54: 234-239.

Cole, S. and Fiorentine, R. 1991. Discrimination against women in science: the confusion of outcome with process. - In: Zuckermann, H. et al. The outer circle. Women in scientific community. Norton and company, pp. 205226.

Colquhoun, D. 2003. Challenging the tyranny of impact factors. - Nature 423: 479.

Csada, R. D. et al. 1996. The "file drawer problem of nonsignificant results" does it apply to biological research? - Oikos 76: 591-593.

Duvall, S. and Tweedie, R. 2000. Trim and Fill: a simple funnel-plot-based method of testing and adjusting for publication bias. - Biometrics 56: 455-463.

Einav, L. and Yariv, L. 2006. What's in a surname? The effects of surname initials on academic success. - J. Econ. Persp. 20: 175-188.

Ford, E. D. 2000. Scientific method for ecological research. - Cambridge Univ. Press.

Friedmann, S. H. and Karlsson, J. O. M. 1997. A novel paradigm. - Nature 385: 480.

Grimm, D. 2005. Suggesting or excluding reviewers can help get your paper published. - Science 309: 1974.

Gurevitch, J. and Hedges, L. V. 1999. Statistical issues in ecological meta analyses. - Ecology 80: 1142-1149.

Jennions, M. D. and Møller, A. P. 2002. Publication bias in ecology and evolution: an empirical assessment using the trim and fill method. - Biol. Rev. 77: 211-222.

Kokko, H. and Sutherland, W. J. 1999. What do impact factors tell us? - Trends Ecol. Evol. 14: 382-384.

Koricheva, J. 2003. Non-significant results in ecology: a burden or a blessing in disguise? - Oikos 102: 397-401.

Kotiaho, J. S. 1999a. Papers vanish in mis-citation black hole. - Nature 398: 19.
Kotiaho, J. S. 1999b. Unfamiliar citations breed mistakes. - Nature 400: 307.

Leimu, R. and Koricheva, J. 2004. Cumulative meta-analysis: a new tool for detection of temporal trends and publication bias in ecology. - Proc. R. Soc. Lond. B 271: 19611966.

Leimu, R. and Koricheva, J. 2005a. Does scientific collaboration increase the impact of ecological articles? - Bioscience 55: 438-443.

Leimu, R. and Koricheva, J. 2005b. What determines the citation frequency of ecological papers? - Trends Ecol. Evol. 20: 28-32.

Loehle, C. 1987. Hypothesis testing in ecology: psychological aspects and the importance of theory maturation. - Q. Rev. Biol. 62: 397-409.

Lortie, C. J. 2000. Why reply (to Hjältén and Price)? - Oikos 90: $185-186$.

Lortie, C. J. and Dyer, A. R. 1999. Over-interpretation: avoiding the stigma of non-significant results. - Oikos 87: $183-184$

Møller, A. P. and Jennions, M. D. 2001. Testing and adjusting for publication bias. - Trends Ecol. Evol. 16: $580-586$.

Nylenna, M. et al. 1994. Multiple blinded reviews of the same two manuscripts. Effects of referee characteristics and publication language. - J. Am. Med. Ass. 272: 149-151.

O'Connor, R. J. 2000. Why ecology lags behind biology. - Scientist 14: 35.

Palmer, A. R. 1999. Detecting publication bias in metaanalyses: a case study of fluctuating asymmetry and sexual selection. - Am. Nat. 154: 220-233.

Schubert, C. and Sinha, G. 2004. A lab of her own. - Nat. Med. 19: $114-115$.

Sears, A. L. W. 2003. Image problems deplete the number of women in academic applicant pools. - J. Women Minorities Sci. Engin. 9: 169-181.

Seglen, P. O. 1994. Causal relationship between article citedness and journal impact. - J. Am. Soc. Inf. Sci. 45: $1-11$.

Song, F. et al. 2000. Publication and related biases. - Health Tech. Assessments 4: 1-115.

Stamp, N. 2003. Theory of plant defensive level: example of process and pitfalls in development of ecological theory. - Oikos 102: 672-678.

Swihart, R. K. et al. 2002. Gray matters in ecology: dynamics of pattern, process, and scientific progress. - Bull. Ecol. Soc. Am. 83: 149-155.

Tomkins, J. L. and Kotiaho, J. S. 2004. Publication bias in meta-analysis: seeing the wood for the trees. - Oikos 104: $194-196$.

Tregenza, T. 1997. Darwin a better name than Wallace? - Nature 385: 480.

Tregenza, T. 2002. Gender bias in the refereeing process? - Trends Ecol. Evol. 17: 349-350.

Tregenza, T. and Wedell, N. 1997. Natural selection bias? - Nature 386: 234.

Van Raan, A. F. J. 1999. Advanced bibliometric methods for the evaluation of universities. - Scientometrics 45: 417423.

Wilkinson, D. M. 1998. Fragments of an entangled bank: do ecologists study most of ecology? - Oikos 82: 393-394. 